\title{
Targeted turbulent structure control in wall-bounded flows via localized heating
}

Cite as: Phys. Fluids 32, 035104 (2020); https://doi.org/10.1063/1.5144387

Submitted: 05 January 2020 . Accepted: 11 February 2020 . Published Online: 03 March 2020

Jean-Pierre Hickey (D), Khaled Younes (D), Matthew X. Yao (D), Duosi Fan (D), and Joseph Mouallem (D)

\section{COLLECTIONS}

Paper published as part of the special topic on Advances in Micro/Nano Fluid Flows: In Memory of Prof. Jason Reese and Collection

Note: This paper is part of the Special Topic on Passive and Active Control of Turbulent Flows.

This paper was selected as Featured

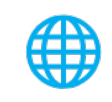

\section{CHALLENGE THE IMPOSSIBLE} WITH OUR PRACTICAL REFERENCE GUIDES

Learn more $\Theta$
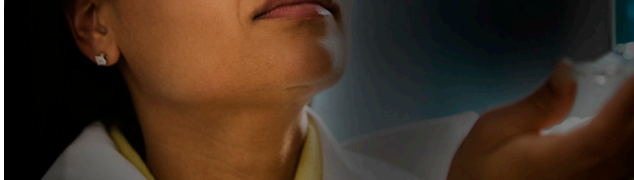


\title{
Targeted turbulent structure control in wall-bounded flows via localized heating
}

Cite as: Phys. Fluids 32, 035104 (2020); doi: 10.1063/1.5144387

Submitted: 5 January 2020 - Accepted: 11 February 2020 •

Published Online: 3 March 2020

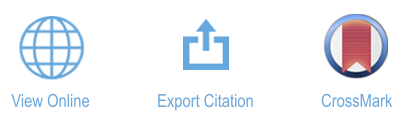

Jean-Pierre Hickey, ${ }^{1, a)}$ (D) Khaled Younes, ${ }^{1}$ (D) Matthew X. Yao, ${ }^{1,2}$ (D) Duosi Fan, ${ }^{\text {(D) }}$ and Joseph Mouallem ${ }^{1}$ (D)

AFFILIATIONS

${ }^{1}$ Department of Mechanical and Mechatronics Engineering, University of Waterloo, Waterloo, Ontario N2L 3G1, Canada

${ }^{2}$ Department of Mechanical and Civil Engineering, California Institute of Technology, Pasadena, California 91125, USA

Note: This paper is part of the Special Topic on Passive and Active Control of Turbulent Flows.

a) Author to whom correspondence should be addressed: j6hickey@uwaterloo.ca

\begin{abstract}
A targeted turbulent flow control strategy, based on selective heating of streamwise-aligned heat strips, is assessed for drag reduction using direct numerical simulations of variable viscosity and compressible turbulent channel flows. As increasing the temperature of a gas increases its viscosity, heating is generally an unfavorable drag mitigation approach. However, through a selective spatial arrangement of the heating array, the slight increase in viscosity and decrease in density can serve to modify the organization of the streamwise-aligned structures and the likelihood of the ejection and sweep events near the wall. This can, under specific conditions, lead to a very modest drag reduction. The optimal spatial arrangement is identified using a bidimensional empirical mode decomposition and targets the near-wall, large-scale turbulent motion. The drag coefficient, at constant mass flow rate, remains unchanged with heating despite up to an $11 \%$ increase in the local viscosity above the heating strips. When accounting for the viscosity variation in the drag reduction calculation, an effective drag reduction of $6 \%$ is observed.
\end{abstract}

Published under license by AIP Publishing. https://doi.org/10.1063/1.5144387

\section{INTRODUCTION}

Coherent vortical structures represent the order within the otherwise disorderly behavior of turbulent flows. The control of these structures remains one of the central tenets to the modern theory of flow control and has been long sought after in many practical applications. For manipulating turbulent coherent structures, either in terms of scale or location, and modifying the structural organization within a turbulent flow offers a direct mechanism to augment mixing, reduce drag, increase lift, suppress noise, or improve heat transfer.

One of the most promising applications for turbulent flow control lies in drag reduction of wall-bounded flows. In this regard, even the slightest reduction in the total shear at the wall can translate into substantial performance gains applicable to various industries, such as aviation and oil and gas. Classically, studies of drag reduction in wall-bounded turbulent flows have centered on two fundamental ideas: $^{1}(1)$ modifying the superficial features of the physical wall and (2) changing the near-wall fluid properties. In either of these two broad categories, active and passive control strategies exist. While active control strategies promise far greater performance gains, the technology needed for their implementation still lacks robustness for engineering applications. As a result, in this work, we focus on a passive approach to turbulent flow control.

By far, the most prominent passive control concept for reducing drag is riblets. ${ }^{2}$ These superficial wall modifications are premised on the idea that localized surface inhomogeneities modify the nearwall turbulent structures, thereby reducing drag. Indeed, a maximum drag reduction of up to $10 \%$ was recorded in experiments for a peak-to-peak riblet spacing of $\sim 15$ wall units. ${ }^{3,4}$ More recent studies have optimized riblet geometry to minimize drag in a laminar boundary layer. ${ }^{5}$ Despite their academic interest, riblets have remained largely out of favor in many engineering applications due to their high cost, weight, and limited range of operation. Several other studies have investigated anisotropic wall porosity, micro-cavities for turbulence attenuation, ${ }^{9}$ and superhydrophobic roughness elements ${ }^{10}$ as well as active approaches such as the use of streamwise aligned plasma actuators ${ }^{11}$ to directly reduce drag or through a spatial arrangement of the pulsatile dielectric barrier discharge array in order to create a Stokes-layer in the flow. ${ }^{12}$ 
On the other hand, changing the near-wall fluid properties provides an attractive alternative to reducing drag. By, for example, application of heated or cooled walls, one can utilize the boundary conditions to alter the near-wall viscosity. Since the shear force in wall-bounded flows is directly related to the viscosity, this provides a direct mechanism to reducing drag. In a gas, a reduction in viscosity can be achieved by application of cooled walls, whereas in a liquid, drag reductions can be realized by heated walls. Recent numerical results obtained by Lee et al. ${ }^{13}$ demonstrated this phenomenon with a $26 \%$ drag reduction, using a wall temperature of $T_{w}=99^{\circ} \mathrm{C}$ and liquid water as the working fluid; a similar observation was noted experimentally. ${ }^{14}$ In their study, Lee et al. ${ }^{13}$ considered an idealized geometry, neglected any buoyancy effects, and found that a lower near-wall viscosity, caused by heating the water, resulted in a decrease in the Reynolds shear stress in the boundary layer. Combined with the fact that turbulence production is reduced and dissipation is enhanced at a lower local Reynolds number, this ultimately yielded a reduction in the skin friction coefficient, $C_{f}$. Using air as the working fluid and including the effects of buoyancy, Kametani and Fukagata ${ }^{15}$ realized a much larger maximum drag reduction of $65 \%$ for uniformly cooled walls.

While the aforementioned drag reductions, particularly that of Kametani and Fukagata, ${ }^{15}$ are encouraging, the authors did not achieve a net energy gain. This severely hampers the prospect of implementation of the control strategy in the real world. Furthermore, it establishes the need for more efficient drag reduction methodologies, a point strongly emphasized by Gad-el-Hak. ${ }^{16}$ Instead of focusing on affecting the entire flow, Gad-el-Hak ${ }^{16}$ proposed a new strategy for attaining efficient drag reductions, dubbed as "targeted control".

In targeted control, the user selectively targets certain coherent structures with the goal of inhibiting or promoting turbulence production, depending on the desired outcome. One of the earliest applications of targeted flow control was the selective suction concept, ${ }^{17-19}$ which used riblets to fix the position of the lowspeed streaks and suction to create an asymptotic turbulent boundary layer. Another example was demonstrated by Yoon et al., ${ }^{20}$ where drag reductions of up to $35 \%$ were attained with heating and cooling strips oriented perpendicular to the channel flow. More recently, other researchers ${ }^{21-23}$ have also used the targeted, spatial heating concept as a means for drag reduction in several flows, including laminar flow. Finally, in an active control setting, Choi et al. ${ }^{24}$ implemented a targeted control strategy to successfully cancel out streamwise vortices.

In this paper, we numerically study a targeted flow control concept, accomplished through the application of streamwise thermal strips oriented parallel to the flow, for a wall-bounded, gaseous turbulent flow. The strips are aligned to impede the large-scale, draginducing motion of the dominant turbulent structures found in the near-wall region. Generally, as previously mentioned, heating of a gaseous channel flow causes an increase in the near-wall viscosity and thus, an expected increase in drag. However, in the present work, we report a modest decrease in drag for an optimal strip alignment. The utilization of the thermal inhomogeneities in the nearwall region may be considered as a net neutral energy system, since waste heat can be recovered and leveraged, especially in the case of flow over aircraft nacelles. In other words, in engineering flows where excess heat is available as a result of aerothermal heating or the proximity to a combustion engine, surface thermal inhomogeneities could be integrated, for example, by varying the thermal conductivity of the surface materials. The paper is organized as follows: Sec. II highlights the numerical details of the simulations, Sec. III discusses the thermal strip alignment, Secs. IV and V report on the impacts of localized heating on the mean flow statistics and turbulence structures, and Sec. VI summarizes the findings and draws the conclusions.

\section{NUMERICAL DETAILS}

\section{A. Computational setup}

We simulate a turbulent channel flow with streamwise-aligned, wall-heated strips as a means of turbulent flow control. The computational domain is shown in Fig. 1 (left). The streamwise, wallnormal, and spanwise directions are defined to be aligned with the $x-, y$-, and $z$-axes, respectively. The simulations are conducted at a nearly incompressible bulk Mach number of 0.2 , and Table I summarizes the main flow parameters and the dimensions of the problem. Here, $R e_{\tau}$ and $R e_{b}$ are the friction and bulk Reynolds numbers, respectively, and the characteristic length is selected as the channel half-height, $h$. A non-slip, isothermal boundary condition with spanwise-varying wall temperature is used on both top and bottom
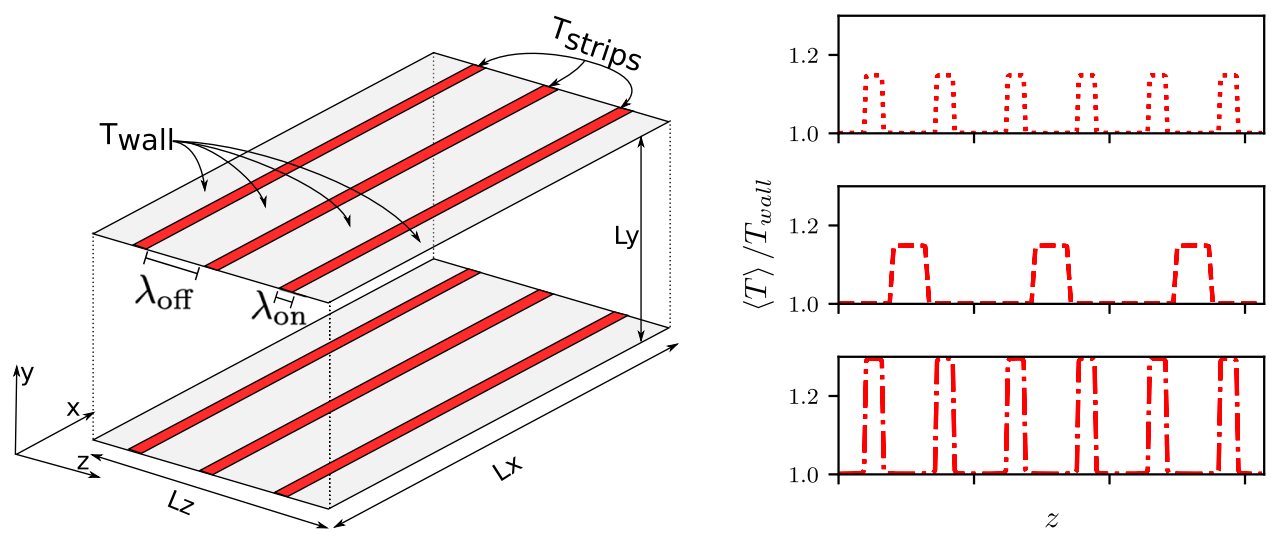

FIG. 1. Schematic diagram of the computational domain, where shaded regions indicate selective heating. 
TABLE I. Common numerical and physical parameters for all the simulations.

\begin{tabular}{ccccc}
\hline \hline$R e_{\tau}$ & $R e_{b}$ & $M_{b}$ & $L_{x} \times L_{y} \times L_{z}$ & $N_{x} \times N_{y} \times N_{z}$ \\
\hline 464 & 8500 & 0.2 & $2 \pi \times 2 \times \pi$ & $216 \times 216 \times 216$ \\
\hline \hline
\end{tabular}

walls. A periodic boundary condition is applied in the streamwise and spanwise directions to reduce the computational cost incurred in reaching a statistically steady turbulent state. The grid is homogeneously distributed in the periodic directions, and a hyperbolic tangent mapping is used in the normal direction to cluster the grid points close to the walls.

The small computational domain is selected based on the recent work of Cheng et al., ${ }^{25}$ where the impact of the domain on the turbulence scales was formally assessed. Based on their results, our domain is deemed appropriate for studying all relevant turbulence scales at the selected Reynolds number. Note that our simulations have a slightly lower $R e_{\tau}$ and a higher total grid count than the authors; ${ }^{25}$ this is done in order to better account for the heat transfer at the walls. Despite the lower $R e_{\tau}$, the turbulent contribution to the drag remains much greater than the laminar viscous term, as will be shown later. Given the small changes arising from the local heating strips, the statistically steady simulations were run for a relatively long time before collecting reliable statistics-the simulations were run to 1500 time units (based on the bulk velocity and channel half-width).

Three cases with different wall heating arrays are simulated (Fig. 1, right), in addition to an unheated case that acts as a comparative benchmark. All the simulations are initialized via a random perturbation to a turbulent mean flow profile. (Since the flow is that of a turbulent channel, the transient memory effects quickly subside, and the flow reaches a statistically steady state after a number of flow through times.) The lateral spacing of the heating strips is varied to impede the large-scale, streamwise-aligned turbulent motion. Primarily, modes 3 and 4, as identified by the bidimensional empirical mode decomposition (BEMD), of the benchmark (unheated) case are targeted; more details are presented in Sec. III. The normalized, isothermal wall temperature, $T_{\text {wall }}$, is chosen to be unity, while the heating strips are set to $T_{\text {strip }} / T_{\text {wall }}=1.15$ for the moderately heated three-strip and six-strip cases (S3 and S6) and $T_{\text {strip }} / T_{\text {wall }}=1.30$ for the strongly heated, six-strip case (HS6). This corresponds to a maximum, normalized change in density of $\Delta \rho / \rho=[15 \%, 30 \%]$ and in viscosity of $\Delta \mu / \mu=[11.1 \%, 21.7 \%]$, respectively. The cases and their definitions are listed in Table II. It is worth mentioning that the strips on both walls are perfectly aligned, uniformly spaced, and cover one third of the total wall area. Subsequently, the only difference between cases S3 and S6 lies in the spatial arrangement of the heating stripsthe average temperature of the walls is identical. Finally, given the streamwise-periodic nature of the channel flow simulations, the bulk momentum of all cases is set to unity.

\section{B. Numerical methods}

The direct numerical simulations (DNS) are performed using the Hybrid code, a high-order, compressible Navier-Stokes solver developed by Larsson ${ }^{26,27}$ and Bermejo-Moreno. ${ }^{28}$ The compressible
TABLE II. Naming convention and heating pattern in the cases studied.

\begin{tabular}{lccccc}
\hline \hline Case name & $T_{\text {strips }} / T_{\text {wall }}$ & $\lambda_{\text {on }}$ & $\lambda_{\text {off }}$ & area & $\Delta x^{+}, \Delta y^{+}, \Delta z^{+}$ \\
\hline Baseline (UH) & $\ldots$ & $\ldots$ & $\ldots$ & $\ldots$ & $(13.5,0.228,6.7509)$ \\
Strips 3 (S3) & 1.15 & $\pi / 9$ & $2 \pi / 9$ & 0.33 & $(13.7,0.2318,6.869)$ \\
$\begin{array}{l}\text { Strips 6 (S6) } \\
\text { Hot strips 6 }\end{array}$ & 1.15 & $\pi / 18$ & $\pi / 9$ & 0.33 & $(13.5,0.227,6.7253)$ \\
(HS6) & 1.3 & $\pi / 18$ & $\pi / 9$ & 0.33 & $(13.5,0.274,6.750)$ \\
\hline \hline
\end{tabular}

Navier-Stokes equations are solved in the conservative form as follows:

$$
\begin{gathered}
\frac{\partial \rho}{\partial t}+\frac{\partial}{\partial x_{j}}\left(\rho u_{j}\right)=0 \\
\frac{\partial \rho u_{i}}{\partial t}+\frac{\partial}{\partial x_{j}}\left(\rho u_{i} u_{j}\right)=\frac{\partial \sigma_{i j}}{\partial x_{j}}, \\
\frac{\partial \rho e_{T}}{\partial t}+\frac{\partial}{\partial x_{j}}\left(\rho e_{T} u_{j}\right)=\frac{\partial u_{i} \sigma_{i j}}{\partial x_{j}}-\frac{\partial q_{j}}{\partial x_{j}},
\end{gathered}
$$

where $\rho$ is the density, $u_{i}$ is the velocity in the $i$-th direction, $e_{T}$ is the total energy (sum of the internal and kinetic energies), and $q_{j}$ is the heat flux. The term, $\sigma_{i j}$ is the normal and shear stress tensor given by:

$$
\sigma_{i j}=-p \delta_{i j}+2 \mu\left(\left(\frac{\partial u_{i}}{\partial x_{j}}+\frac{\partial u_{j}}{\partial x_{i}}\right)-\frac{\delta_{i j}}{3} \frac{\partial u_{k}}{\partial x_{k}}\right),
$$

where $p$ is the thermodynamic pressure, $\mu$ is the dynamic viscosity, and $\delta_{i j}$ is the Kronecker delta. The thermodynamic variables are coupled through the ideal gas equation and Fourier's law is used to compute the heat flux in the energy equation [Eq. (3)]. To account for temperature changes, a power-law for viscosity is used; this takes the form of $\mu=\mu_{r e f}\left(T / T_{r e f}\right)^{3 / 4}$, where $\mu_{\text {ref }}$ is set based on the desired Reynolds number of the flow. The power-law viscosity profile remains very similar to Sutherland's law, but it has the advantage of being more computationally efficient.

The governing Eqs. (1)-(3) are solved in Hybrid in the nondimensional form, on a structured Cartesian grid. A sixth-order central difference scheme is used to discretize the spatial derivatives; this is supplemented with high-order artificial dissipation to maintain numerical stability and computational efficiency. A fourthorder Runge-Kutta scheme is used for the time integration. The solver has been rigorously validated in the case of shock-turbulence interactions ${ }^{27}$ and wall-bounded flows, ${ }^{29}$ and the accuracy of the numerical schemes is well established.

\section{THERMAL STRIP ALIGNMENT}

To locally modify the thermodynamic (temperature, density) and thermophysical (viscosity) properties of the fluid, we use streamwise-aligned heating strips (Fig. 1, left). The strips are to be arranged such that the near-wall turbulence regeneration mechanism is impeded. To this end, BEMD is first performed on the benchmark case to identify the large-scale motion in the flow. The energy 


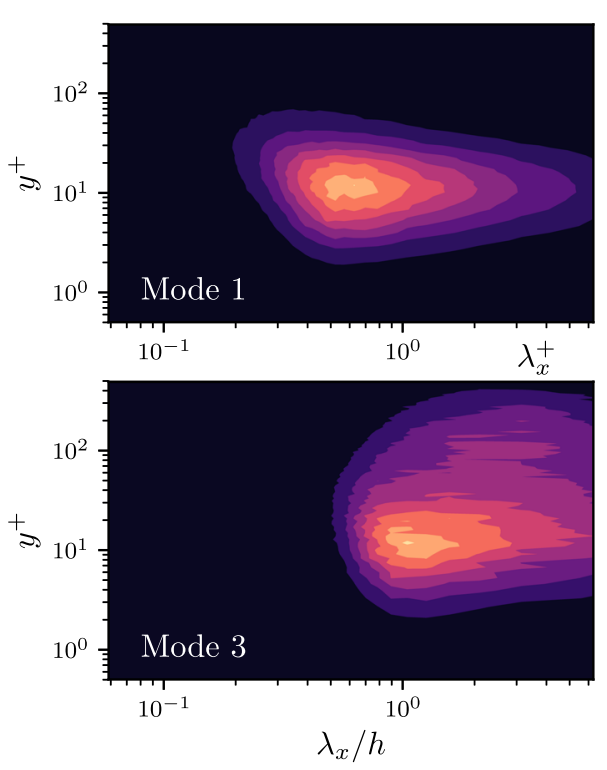

contained in each of the modes is shown in Figs. 2 and 3. The reader is referred to 25 and 31 for details on the implementation of the BEMD and its underlying theory. Second, from Fig. 3, it is evident that the large-scale motion (mode 3 ) in the channel has a characteristic spanwise spacing of $\sim 0.8 h$. This corresponds to a quarter of the total spanwise domain. Since this large-scale motion is believed to be responsible for sustaining the small-scale, shear-inducing turbulent flow near the wall, ${ }^{32}$ the spacing of the strips is designed to be $\lambda_{z}=\pi / 6$ and $\lambda_{z}=\pi / 3$, corresponding to the six-strip arrangement (S6 and HS6), as well as the three-strip arrangement (S3). Note that in Figs. 2 and 3, modes 1 and 2 are scaled with the inner units while modes 3 and 4 are scaled with the channel half width.
We study two different isothermal heating values of $T_{\text {strip }} / T_{\text {wall }}$ $=1.15$ (S3 and S6) and 1.3 (HS6). The underlying drag reduction strategy was to minimally heat the base flow (which, recall, results in an increased viscosity), while locally inhibiting the preferential separation between the streamwise-aligned, large-scale motion. As such, the bulk heating in the channel is increased by about $4.5 \%$ for the moderately heated cases (S3 and S6) and 7.5\% for the strongly heated case (HS6). The resulting bulk viscosity is increased by $3.3 \%$ and $5.6 \%$, respectively, while the local viscosity on the strips is higher by $11.1 \%$ and $21.7 \%$. As the bulk momentum of the channel flow is set to unity due to the selected non-dimensionalization, we expect an increase in drag, proportional to the increase in bulk viscosity.
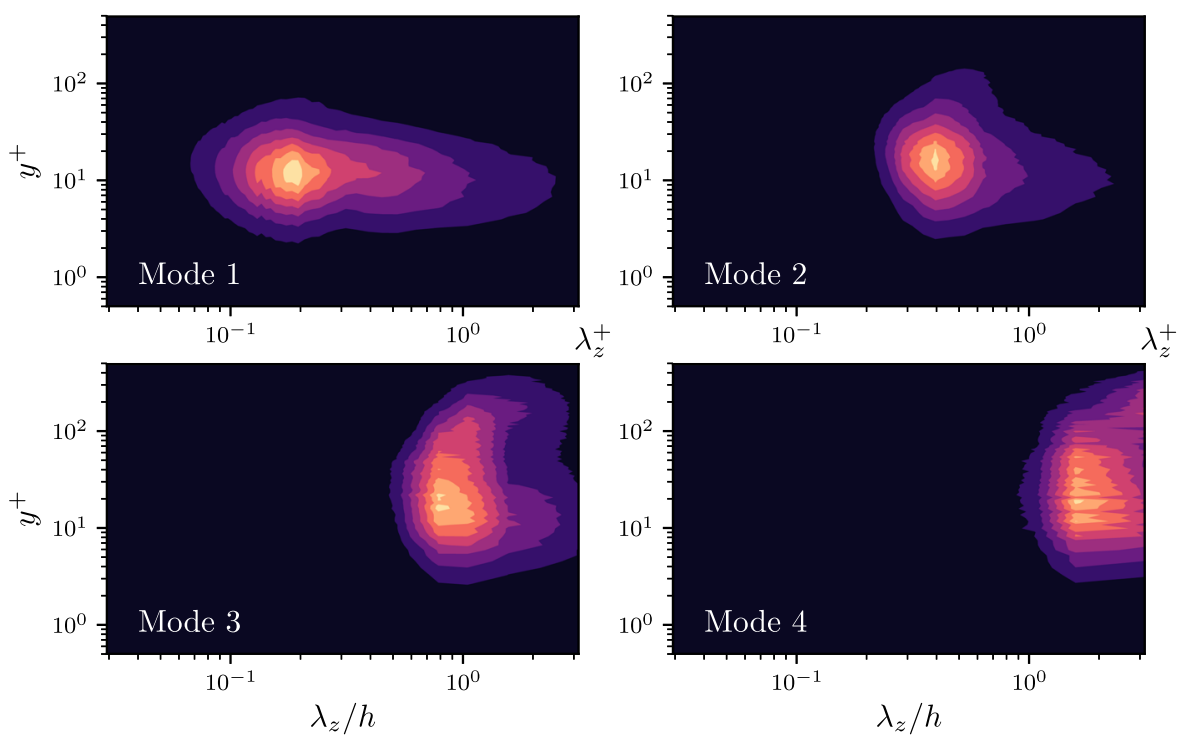

FIG. 3. Spanwise, premultiplied spectrum of all four modes of $u^{\prime}$ for the baseline (unheated) case. Note that the spanwise distance is scaled with inner wall units for modes 1 and 2 and with the channel half-height for modes 3 and 4 . 


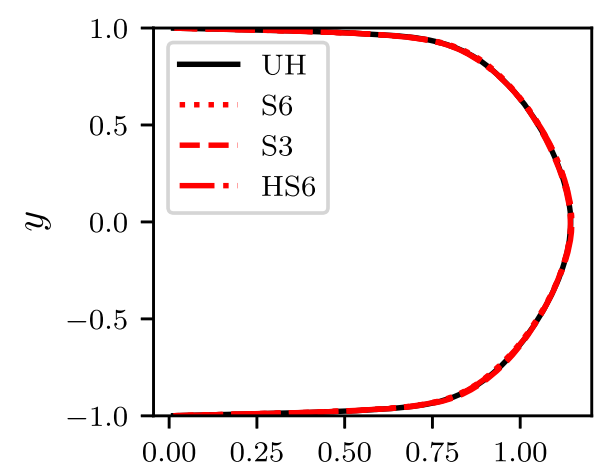

$\langle u\rangle$

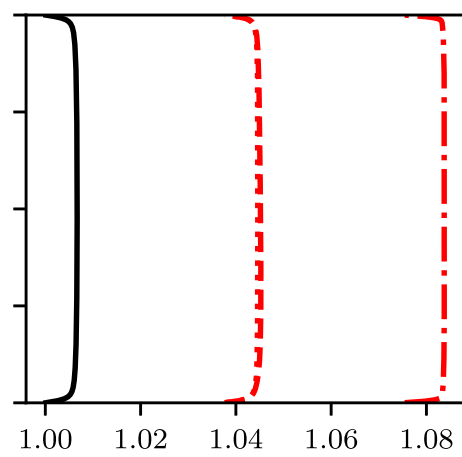

$\langle T\rangle / T_{\text {wall }}$

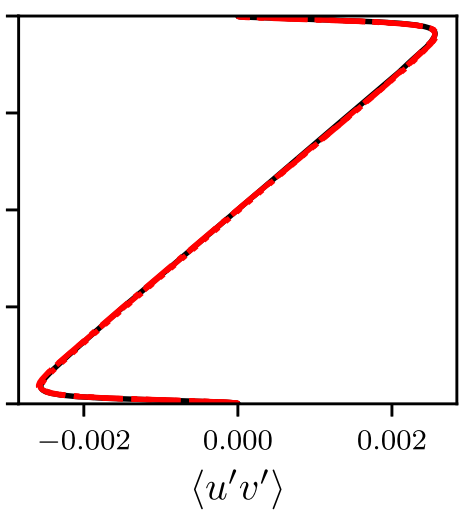

FIG. 4. Averaged streamwise velocity, temperature, and Reynolds stress, $\left\langle u^{\prime} v^{\prime}\right\rangle$, for all cases.

\section{TURBULENCE STATISTICS}

Given the very slight thermodynamic and thermophysical variations caused by the local, inhomogeneous wall heating, we detail our systematic approach in conducting the simulations followed by an analysis, from an integral perspective of the turbulence statistics. First, all simulations are run until a statistically steady state is reached (more than 200 flow through times). Then, the data are collected for every two characteristic time units of the flow (based on the channel half-height and bulk streamwise velocity). Finally, all the statistics are compiled using at least 100, three-dimensional, statistically de-correlated snapshots. The statistical convergence was carefully assessed by evaluating the fluctuations in time of the wall shear stress and other higher-order statistics (see Appendix).

\section{A. Bulk statistics}

We now show that the impact of the targeted heating on the bulk flow remains minimal. Recall that the local thermophysical variation due to heating is small and that the heating is only applied to a third of the total wall area. Here, the averaging, denoted by angle brackets \langle\rangle , is done over the periodic directions ( $x$ and $z$ ) and over all collected snapshots. Figure 4 (left) shows the average streamwise velocity, $\langle u\rangle$. As can be seen, it remains nearly invariant to the heating (we will show later that each case will admit a slightly different velocity gradient at the wall). A very slight increase in the bulk temperature of the flow is observed, Fig. 4 (center), and as expected, the cases with the same isothermal conditions but different spatial arrangements (S3 and S6) yield an identical bulk temperature. The Reynolds stresses of all cases show nearly overlapping profiles (Fig. 4, right). Similarly, a focused view on the normalized, near-wall mean, and turbulent properties reveals no significant changes due to the targeted heating (Fig. 5). It is worth mentioning that we confirmed these results by randomly sub-sampling snapshots from our dataset and verifying that the observed trends are symmetric on each wall.

\section{B. Conditional statistics}

As the impact of the inhomogeneous surface heating cannot be observed in the mean flow or in the bulk flow turbulence statistics, conditional averaging is used in this section to highlight the effects of heating on the flow. Here, the statistics are conditioned separately on the $z$-locations with and without wall heating.
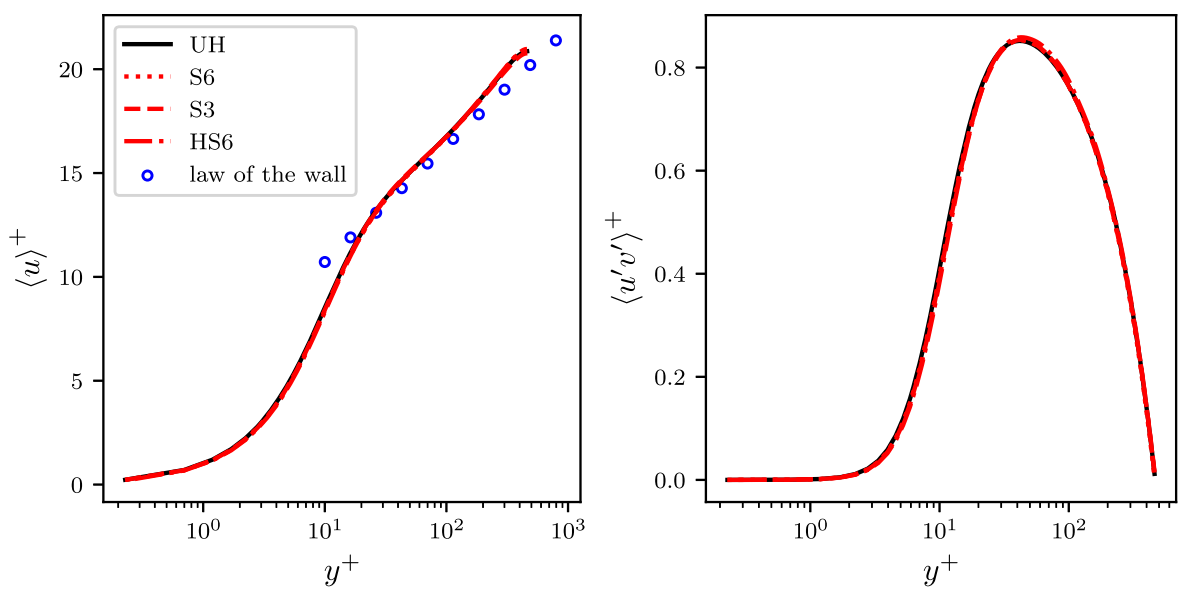

FIG. 5. Near-wall velocity scaling (left) and normalized Reynolds stresses (right) for all cases. 

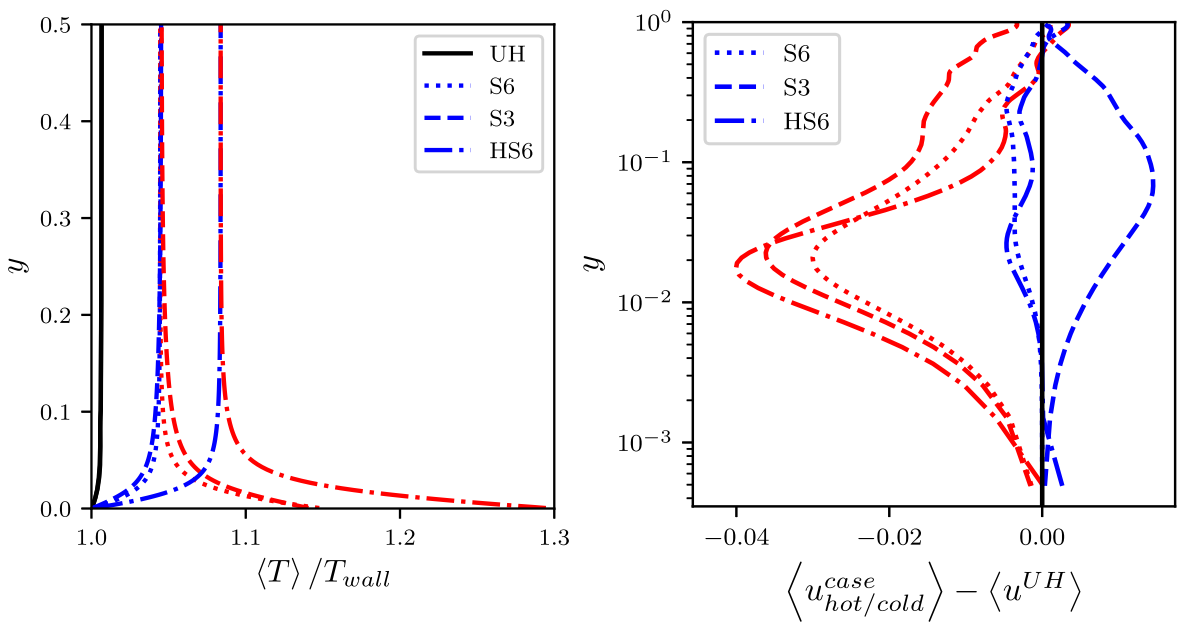

FIG. 6. Conditional mean temperature distribution (left) and streamwise velocity difference between the heated and unheated, baseline case (right) as a function of the normalized distance from the wall. Averages above the heated strips are shown in red and in blue for the unheated regions.

The conditional temperature distribution for all cases is shown in Fig. 6 (left), and it is observed that the mean temperature only differs appreciably in the bottom sixth of the channel, below $y / h<0.16$. It is also noted that the influence of the larger heating strips in the S3 case extends deeper into the channel (at larger heights). This can be attributed to the reduced mixing obtained between the unheated and heated regions in the S3 case; more mixing is expected in S6 and HS6 because of the larger number of strips.
Similarly, when looking at the conditional mean velocity profiles in Fig. 7, we observe that the streamwise velocity profile in the heated regions of the domain always falls below the unheated profile. The difference is seen throughout much of the domain, especially in the viscous sub-layer and buffer region, and it is further highlighted by Fig. 6 (right), which shows that the largest difference occurs near the wall, at $y \sim 0.025 h$.

To better understand the effects of heating on the nearwall turbulence events, quadrant analysis of the streamwise and
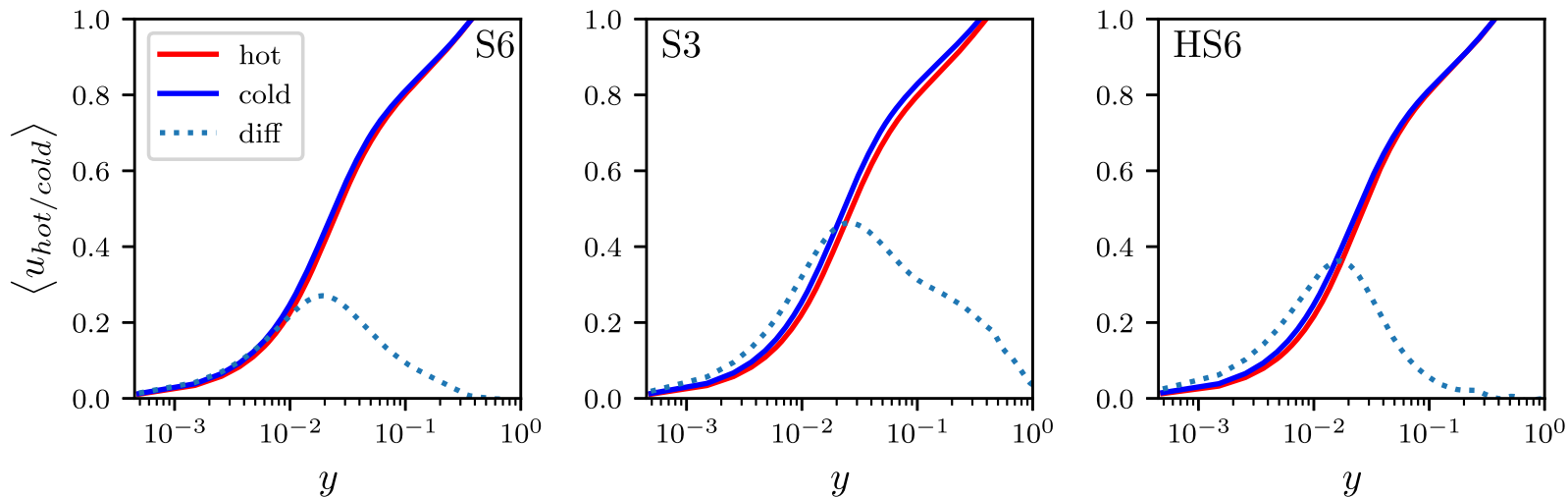

FIG. 7. Conditional mean streamwise velocity. Averages above the heated strips are shown in red and in blue for the unheated regions. Dotted line: the difference (diff) between the cold (unheated) and hot (heated) conditional profiles (multiplied by 10 to highlight the changes).

TABLE III. Probability distribution of the quadrants of $u^{\prime}$ and $v^{\prime}$, conditioned on the local heating of the wall at each $x$ - and $z$-location. The analysis is done at $y^{+}=45$. The value in parenthesis corresponds to the difference between the hot and cold conditional probability.

\begin{tabular}{lcccc}
\hline \hline Case & UH (\%) & S6 (hot/cold) & S3 (hot/cold) & HS6 (hot/cold) \\
\hline Q1 and Q3 & 35.6 & $35.3 \% / 35.2 \%(+0.1 \%)$ & $36.0 \% / 34.9 \%(+1.1 \%)$ & $35.3 \% / 35.2 \%(+0.1 \%)$ \\
Q2 (ejection) & 30.2 & $31.5 \% / 28.7 \%(+2.8 \%)$ & $34.7 \% / 27.3 \%(+7.4 \%)$ & $30.3 \% / 29.0 \%(+1.3 \%)$ \\
Q4 (sweep) & 34.1 & $33.2 \% / 36 \%(-2.8 \%)$ & $29.3 \% / 37.8 \%(-8.5 \%)$ & $34.4 \% / 35.9 \%(-1.5 \%)$ \\
\hline \hline
\end{tabular}



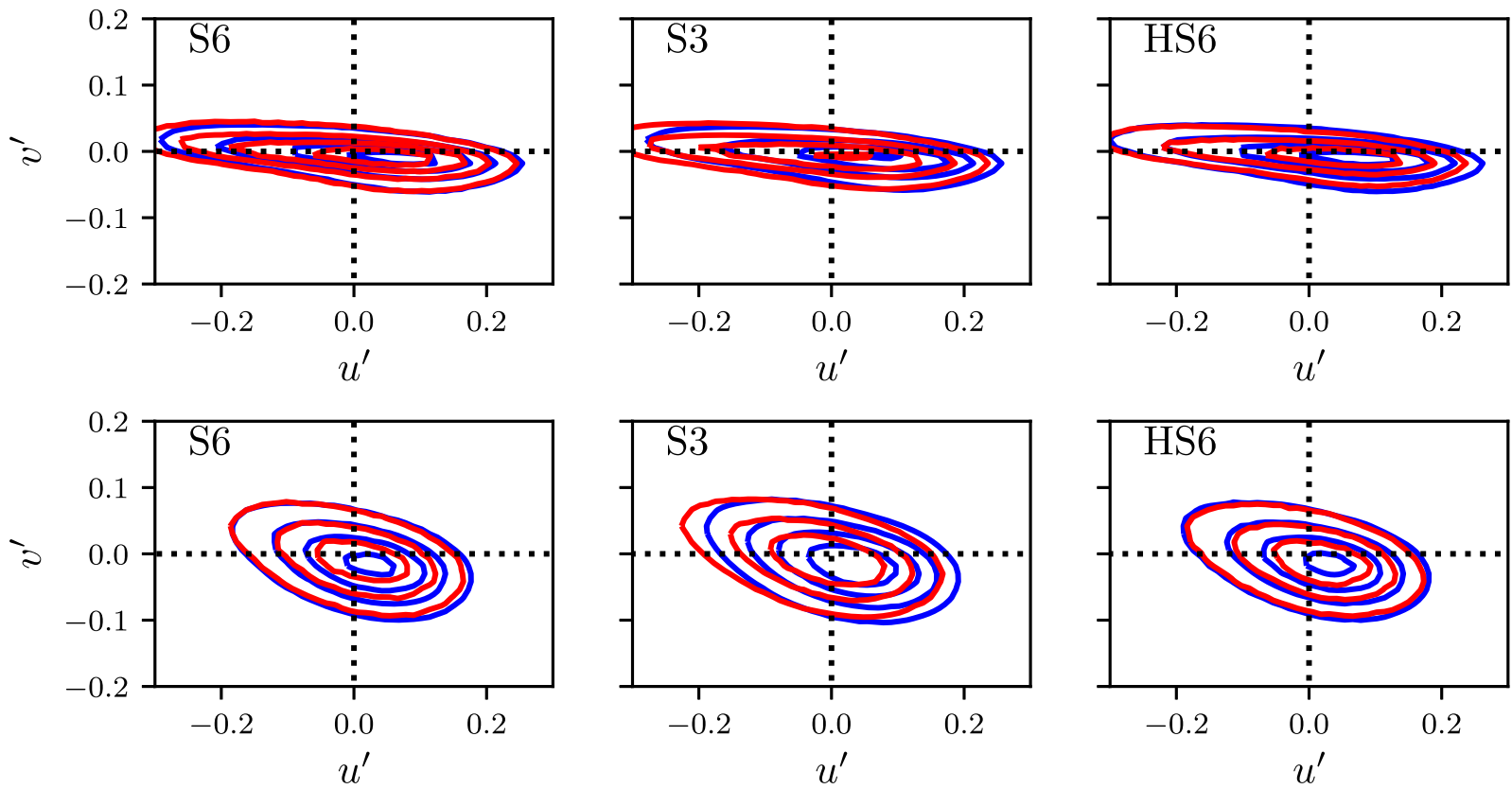

FIG. 8. Quadrant analysis on $u^{\prime}$ and $v^{\prime}$ at $y^{+}=20$ (top row) and $y^{+}=45$ (bottom row). Results are conditioned based on the local wall heating, red: heated region and blue: unheated region. The velocity perturbations are not normalized.

wall-normal fluctuations is performed. Table III summarizes the conditional probability of the turbulence events in the early log-law region of the boundary layer, at $y^{+}=45$; Fig. 8 shows the results schematically at two locations in the boundary layer. As expected, due to the lower density and higher viscosity, a notable increase in the probability of ejection events and a decrease in the sweep events are noted above the heated portion of the wall. The same trend is also observed at other wall-normal locations, at $y^{+}=9$ and 20. In all
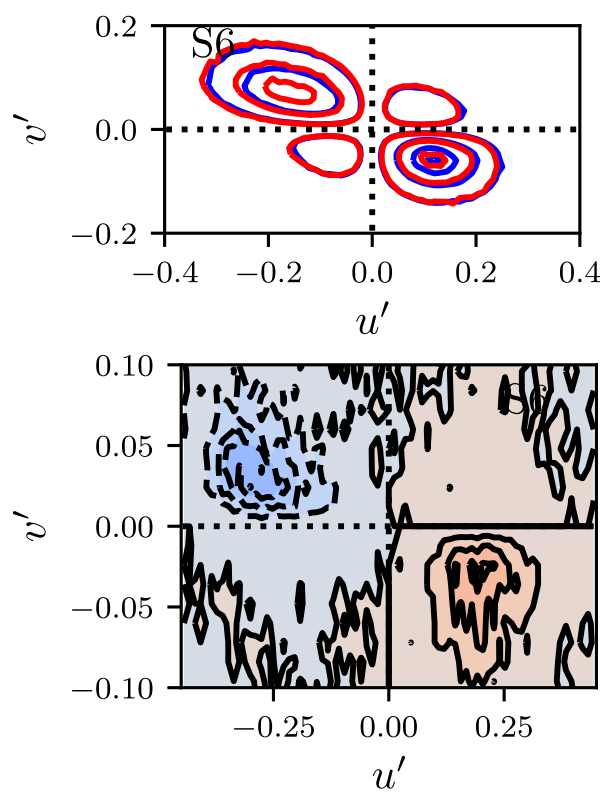
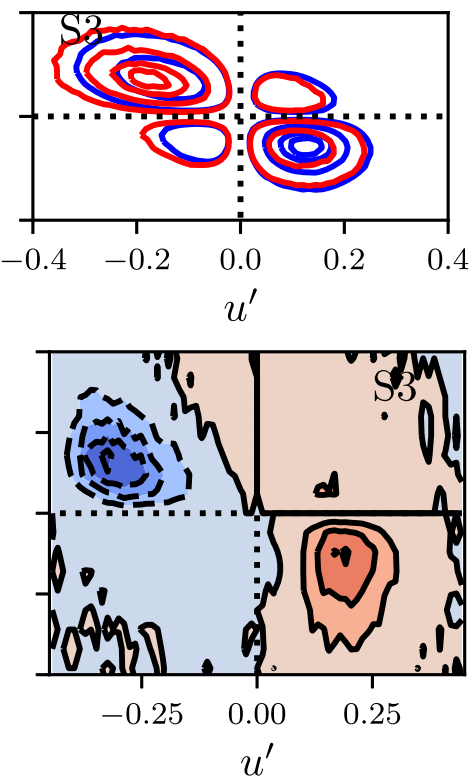
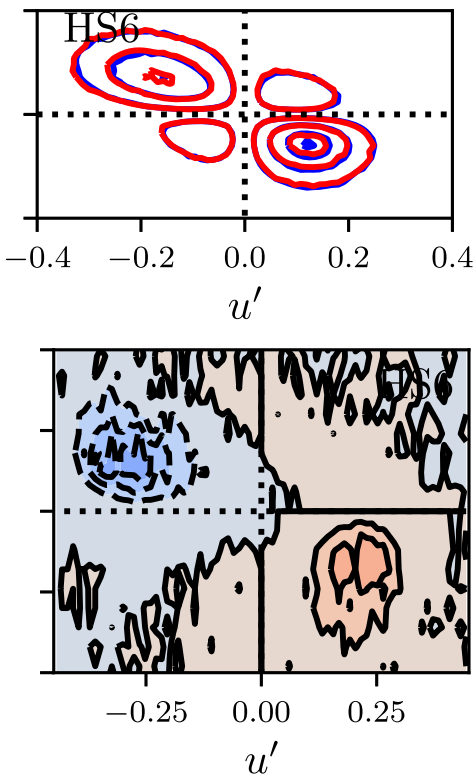

FIG. 9. Covariance integrand of $u^{\prime}$ and $v^{\prime}$ conditioned on the local wall heating at $y^{+}=20$. Top: overlapped covariance integrands of the heated region (red) and unheated (blue) region. Bottom: difference between the heated and unheated covariance integrands. Contour lines are at $0.25,0.75,1.25$, and $1.5\left(\times 10^{-5}\right)$. The velocity perturbations are not normalized. 
the cases, however, the change in Q1 and Q3 events remains modest; the most significant changes occur for the $\mathrm{S} 3$ case.

The covariance integrand, defined as $u^{\prime} v^{\prime} P\left(u^{\prime}, v^{\prime}\right)$, where $P\left(u^{\prime}\right.$, $\left.v^{\prime}\right)$ is the probability density of the $u^{\prime}$ and $v^{\prime}$ events, provides additional insight into the magnitude of the turbulence events (Fig. 9). The results provide the same general observations as the probability distribution, but we observe a clear shift in the peak of the covariance integrand, between the heated and unheated regions of the domain (Fig. 9, top); this is further emphasized by the difference between the two covariance integrands, shown in Fig. 9 (bottom). Interestingly, the heated region shows a significant increase in ejection (Q2) events, evident by the negative value of the difference between the unheated and heated covariance integrands, yet this is accompanied by an increase in the sweep events over the unheated parts of the domain. Therefore, in order to achieve an overall drag reduction, the benefits of local heating must overcome the detrimental contributions of the unheated portions of the domain.

\section{FLOW CONTROL AND DRAG REDUCTION}

While the quadrant analyses provide a statistical measure determining the likelihood of occurrence of certain turbulence events in a given flow, our aim in this work is to selectively target the coherent structures found in wall-bounded turbulent flows and potentially realize a drag reduction. To this end, the impact of local heating is investigated first in terms of structural changes to the flow and then formally quantified by breaking down the contributions to the skin friction.

\section{A. Turbulence structures}

To start, we study the structural changes using BEMD. The premultiplied spectra of the spanwise and streamwise velocity fluctuations, obtained by performing BEMD on all the cases studied are shown in Figs. 10 and 11. When comparing the energy distribution among the modes, we observe somewhat surprisingly very little differences. Although we note slight changes in the higher modes of the spanwise premultiplied spectrum (Fig. 11), in general, at least spectrally, local heating does not appear to impact the size or the organization of the main turbulence structures in the flow. Similarly, the overall turbulent kinetic energy spectra remain nearly identical for all the cases studied (results not shown).

Then, to better understand the spatial organization of the largescale turbulence structures and their impact on the near-wall shear, the Reynolds stresses are conditionally sampled. Here, the sampling is based on the intensity of the local Reynolds stress, and we only average the stresses whose magnitude is greater than one standard deviation from the mean. Mathematically, this can be written as: $\left\langle\left[u^{\prime} v^{\prime}(x, y, z)\right] \mid \operatorname{abs}\left[u^{\prime} v^{\prime}(x, y, z)\right]>\sigma\left(u^{\prime} v^{\prime}\right)\right\rangle$. The approach followed herein thus allows us to isolate the significant turbulence stress events. These, in turn, are related to the large-scale motions in the channel. Figure 12 shows the results of such averaging in the streamwise direction. It is evident that the overall structural organization in all the cases remains approximately the same, with two pairs of large-scale structures identified on each wall. The spanwise wavelength of the structures is calculated to be $\Delta z \approx 0.8 h$. Recall that this is identical to the width of mode 3 from the unheated BEMD analysis (Fig. 3).

While the spanwise spacing of the structures does not significantly change, the preferential location of these events is influenced by the local heating strips. As the color scaling is identical in all the subplots of Fig. 12, it can be concluded that the structural coherence is enhanced for the strongly heated case (HS6), whereas the moderately heated cases show an approximately similar level of coherence. Based on those observations, it is clear that heating, or more precisely the local change in viscosity and density, impacts the relative position of the large-scale structures in the channel flow, without necessarily altering their average spacing. As a result, local heating can be utilized to directly control the spatial organization of the large-scale turbulence structures.
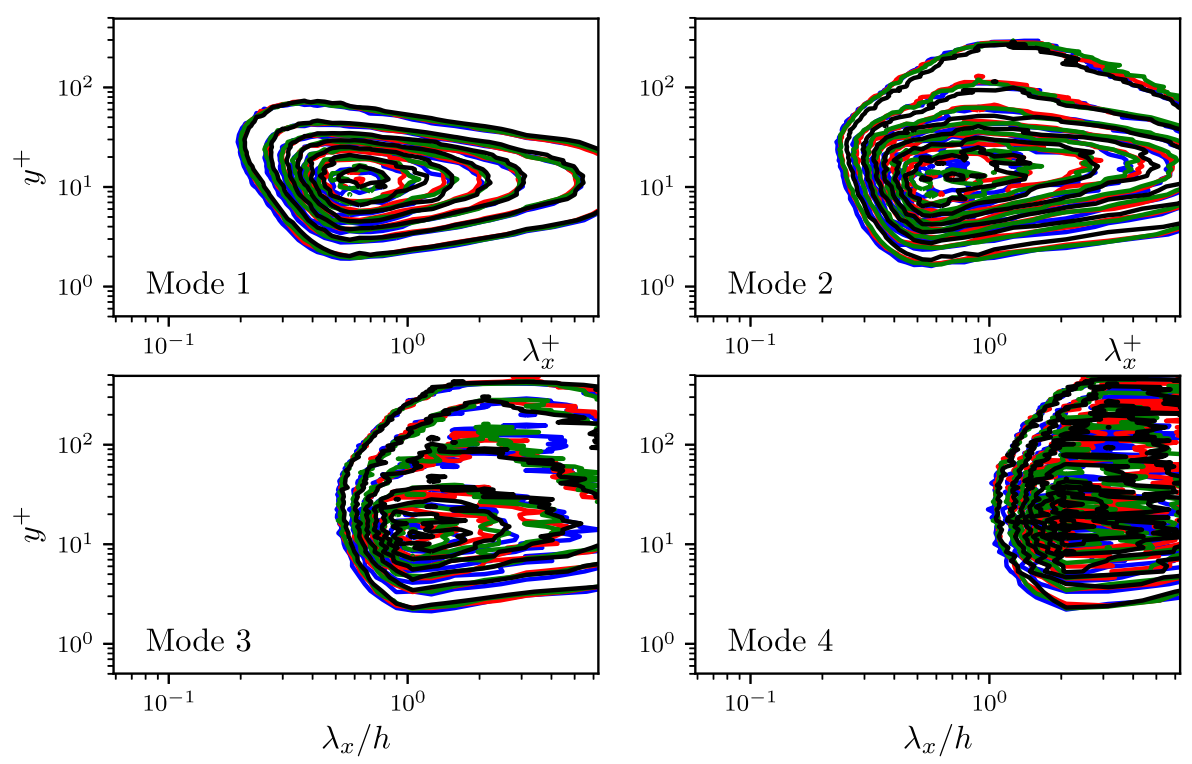

FIG. 10. Streamwise, premultiplied spectrum of all four modes of $u^{\prime}$ for the $\mathrm{UH}$ (blue), S3 (green), S6 (red), and HS6 (black) cases. Note that the streamwise distance is scaled with inner wall units for modes 1 and 2 and with the channel half-height for modes 3 and 4 . 

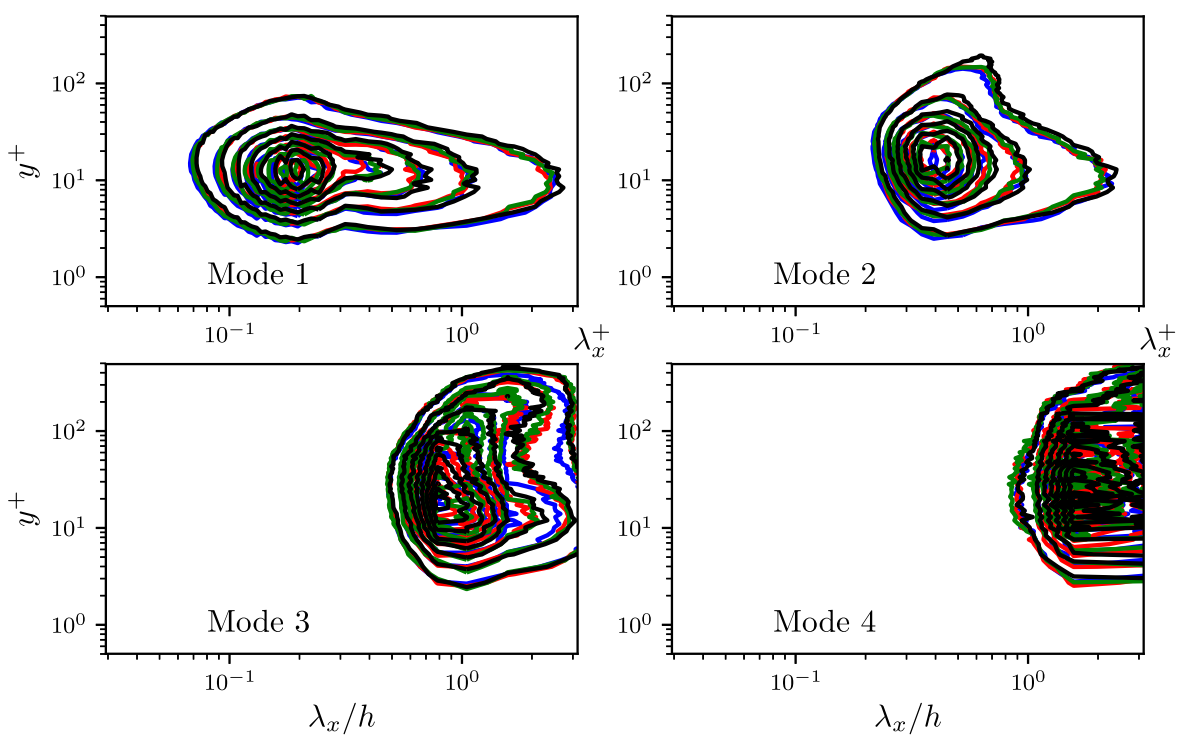

FIG. 11. Spanwise, premultiplied spectrum of all four modes of $u^{\prime}$ for the UH (blue), S3 (green), S6 (red), and HS6 (black) cases. Note that the spanwise distance is scaled with inner wall units for modes 1 and 2 and with the channel half-height for modes 3 and 4 .
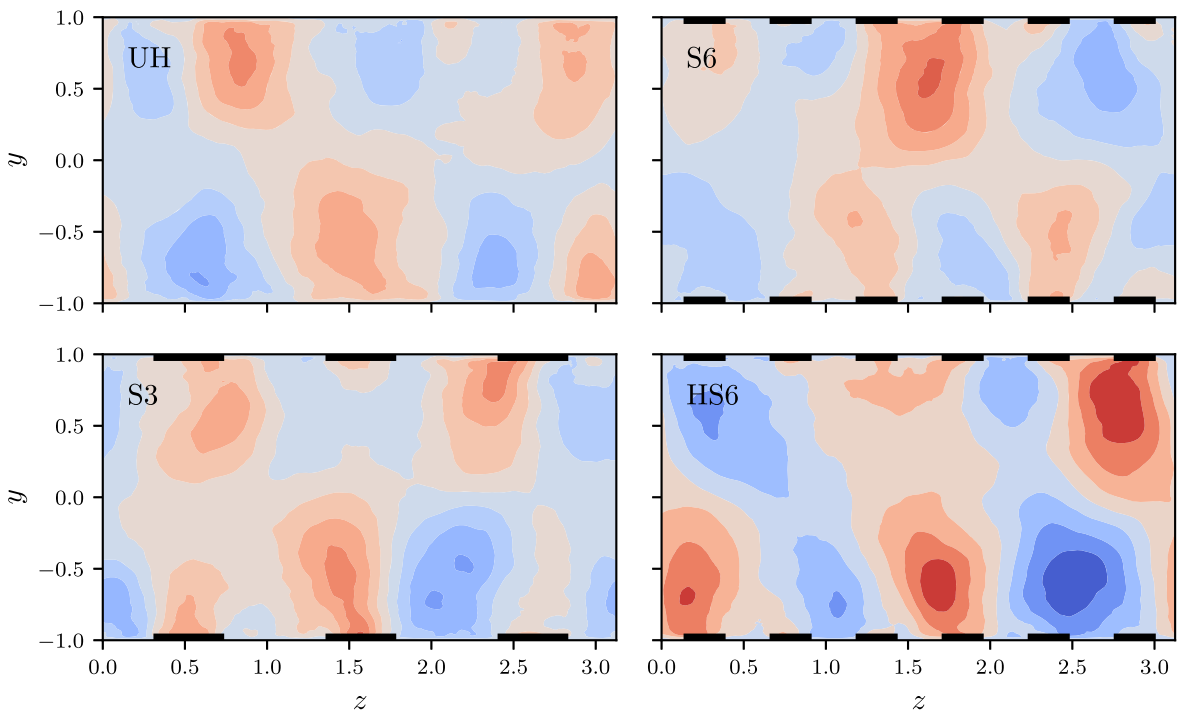

FIG. 12. Streamwise-averaged conditional statistics of $\left\langle u^{\prime} v^{\prime}\right\rangle$. Only turbulence events with a magnitude greater than one standard deviation from the mean are averaged. Thick black lines indicate the location of the wall heating.

To highlight the impact of the heating strips on the largescale structures, a phase-averaged velocity-averaged over one heating strip wavelength-is computed and plotted. Figure 13 shows velocity, which explains the increase in Q2 events observed in Table III, that the lift-up is sustained by two streamwise-aligned, counter-rotating rollers on either side of the heating strip. Natuthat the heating strips cause a lift-up, or a positive wall-normal rally, these rollers are also responsible for the increased sweep (Q4)
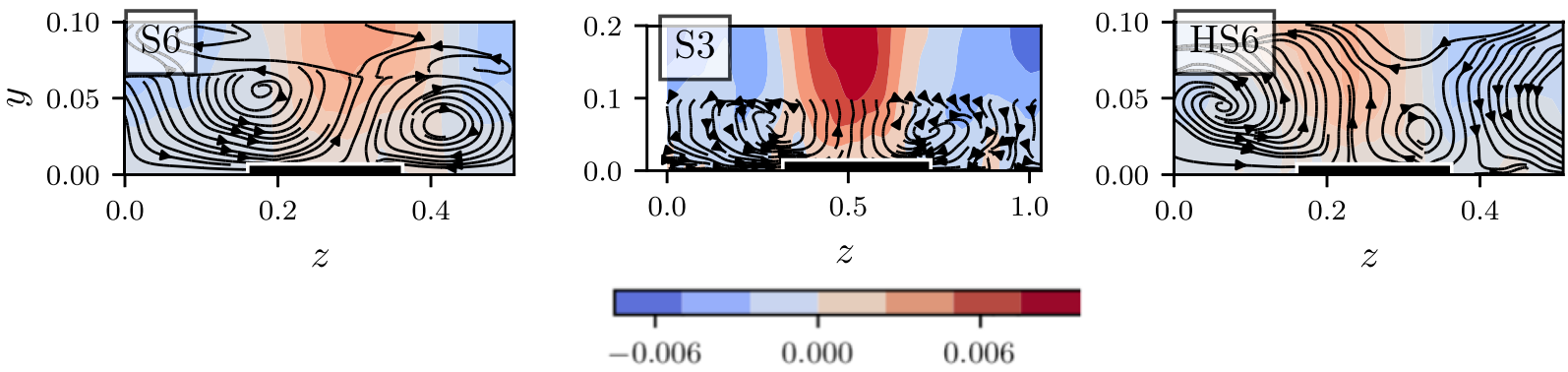

FIG. 13. Streamlines of the phase-averaged mean velocity in the yz plane. Colored contours correspond to the phase-averaged, wall-normal velocity. 
events over the unheated regions of the domain. Figure 14 shows the impact of these near-wall structures on the ensemble-averaged, streamwise velocity fluctuations. Above the heating strips, slower streaks with negative velocity fluctuations are observed, whereas above the unheated regions, faster streaks are seen (Fig. 14, top). Far away from the wall, these structural changes are expectedly lost (Fig. 14, bottom).

The near-wall structural organization can be further inferred from two-point correlations of the kinematic quantities. These are
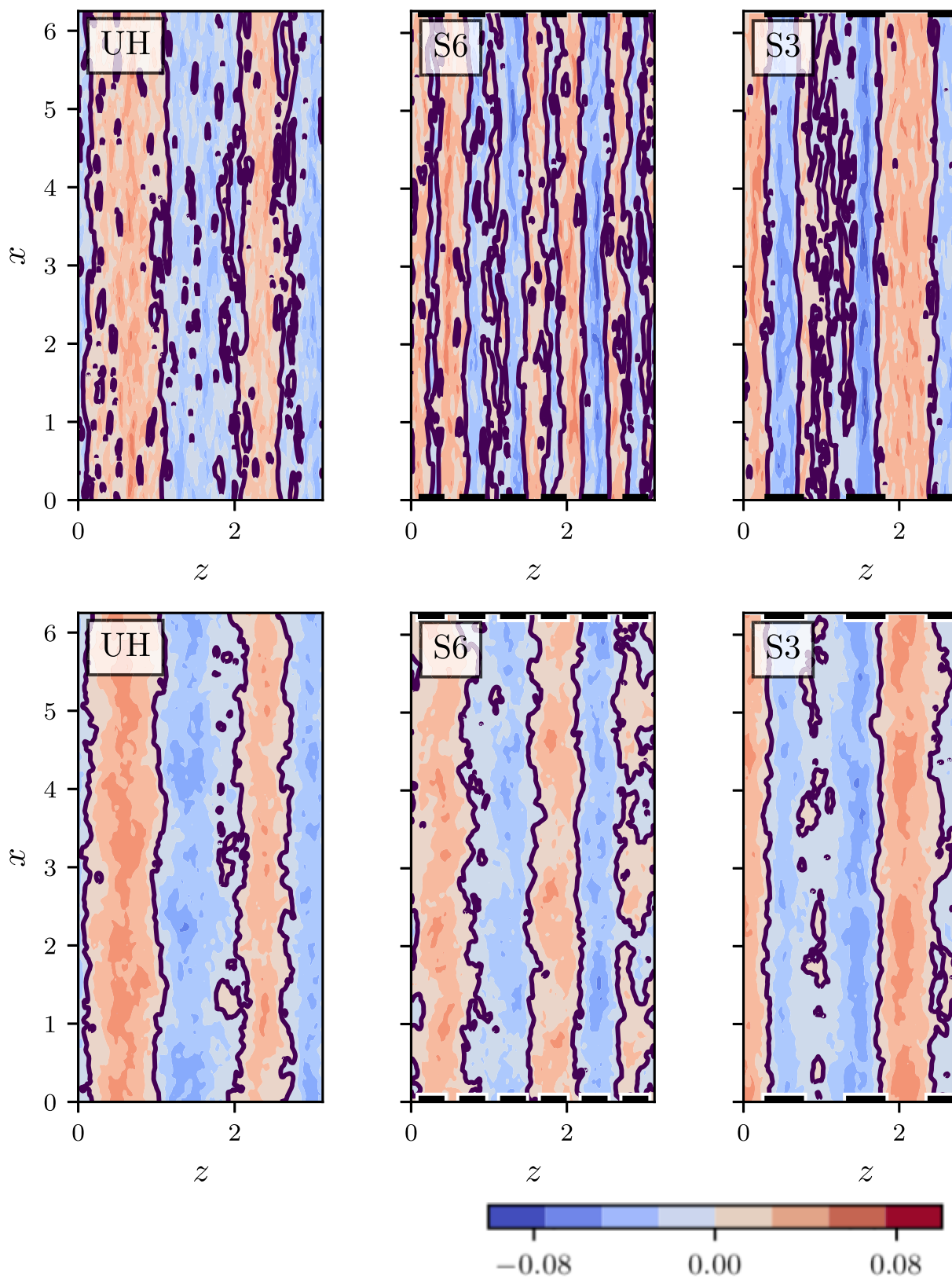

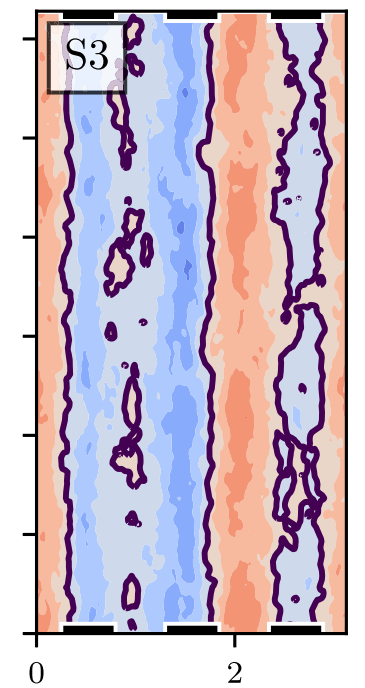

calculated for the streamwise velocity, at each wall-normal location, and presented in Fig. 15. A rather significant change is noted among the cases, with a large reduction of the streamwise correlation length in the S6 case and a slight increase in the length for S3 and HS6. At $y^{+}=20$, Fig. 16 (center) shows that the spanwise correlation length is reduced for all the heated cases. Similarly, when comparing the twopoint correlations of density along the spanwise direction (Fig. 16, right), a decrease in the correlation lengths is noted only for the S6 and HS6 cases; the three-strip (S3) case yields a much longer
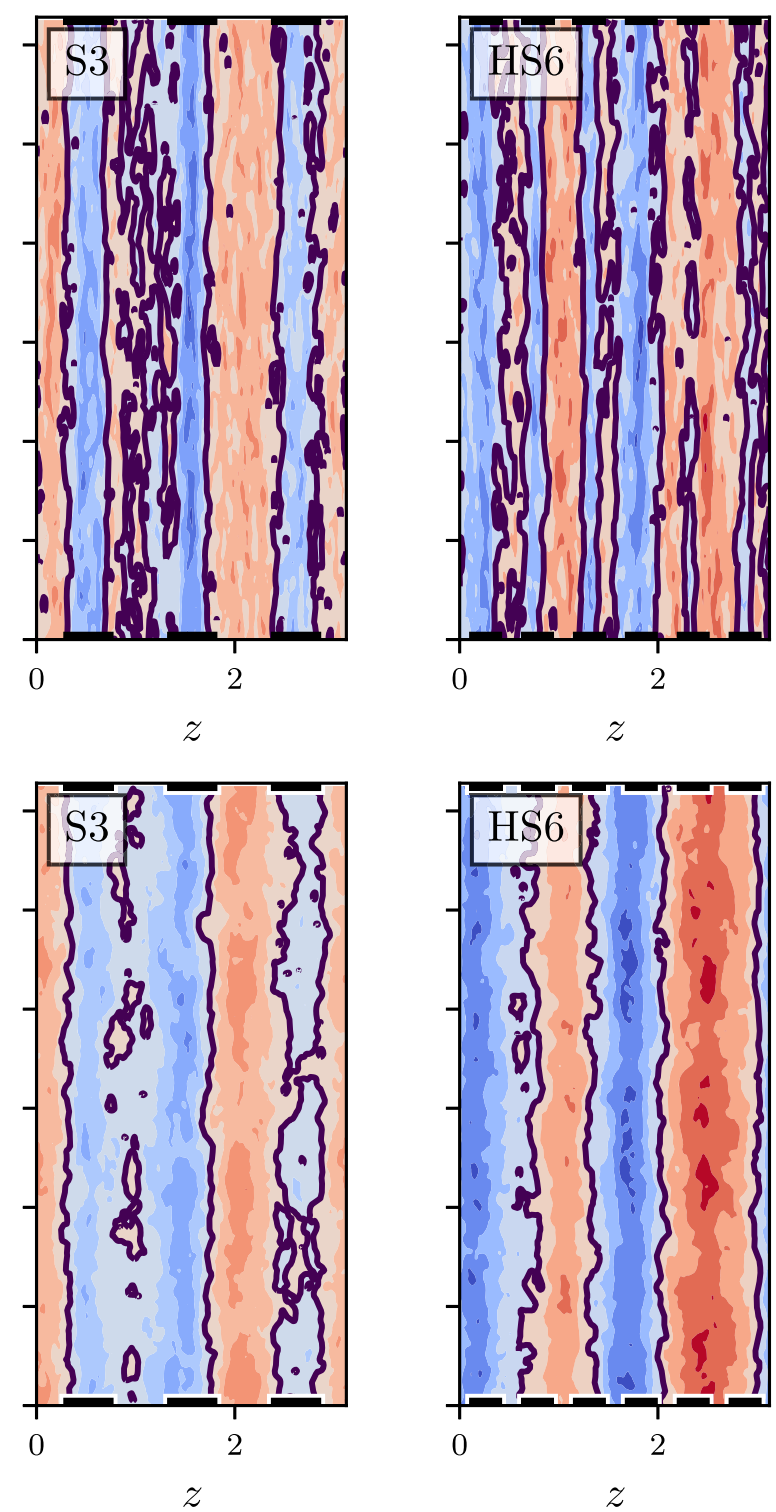

0.08

FIG. 14. Ensemble-averaged, streamwise velocity fluctuations at $y / h=0.02$ (top) and $y / h=0.28$ (bottom). Red regions indicate high-speed streamwise velocity fluctuations, whereas blue regions are for low-speed streaks. Thick black lines indicate the location of the heating strips. 

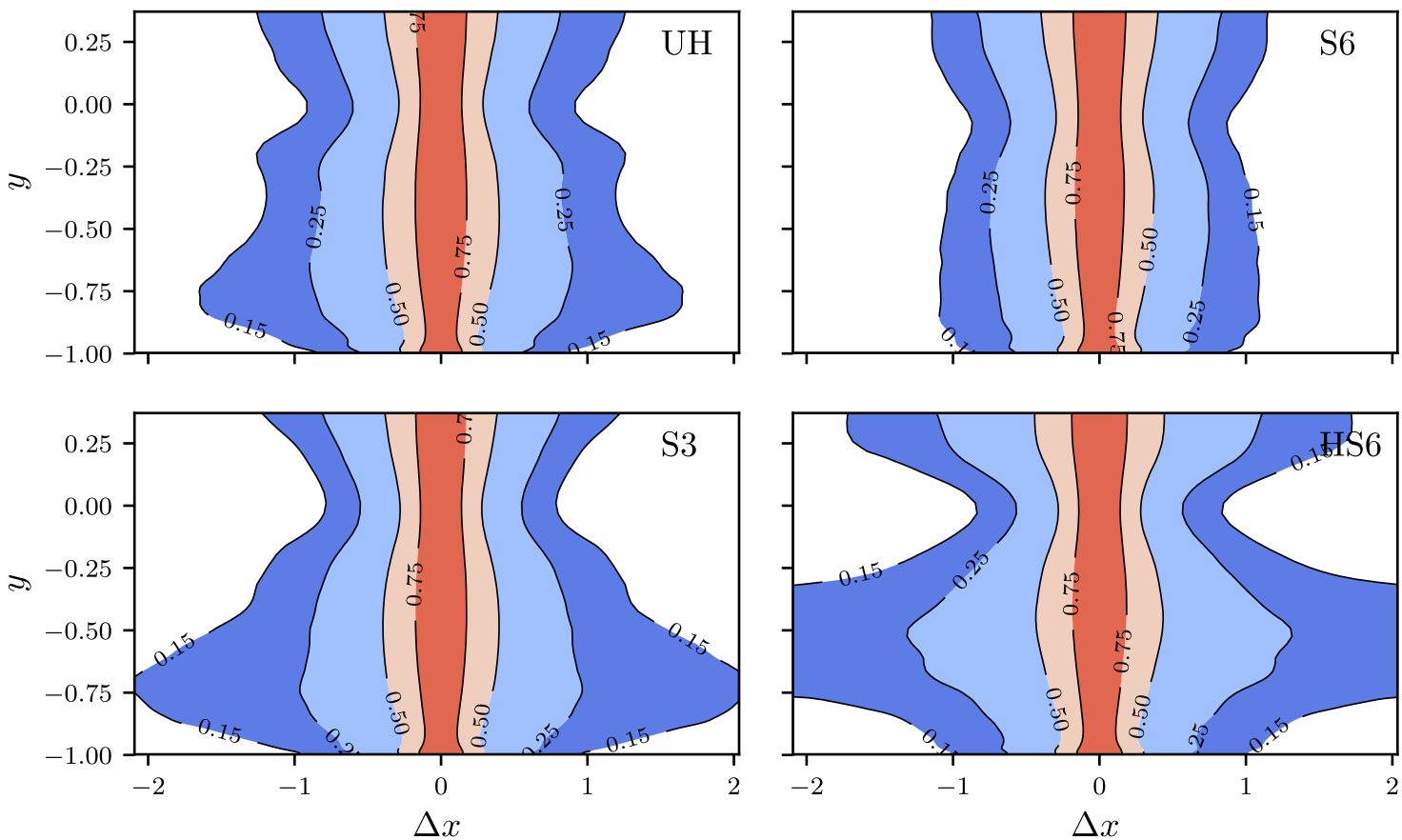

FIG. 15. Two-point correlations of streamwise velocity in the streamwise direction; values are normalized by Reynolds stress $\left\langle u^{\prime} v^{\prime}\right\rangle$ at each $y$ location.

correlation distance (we observe a non-zero correlation over a significant spanwise distance).

As the local heating was shown to impact the ejection and sweep events and modify the spatial organization of the nearwall structures, the spatial correlation of the velocity-temperature fluctuation, $\left\langle u^{\prime} T^{\prime}\right\rangle$, is evaluated. Since the lower local density above the heated strips causes additional ejection events, pushing the low velocity $\left(u^{\prime}<0\right)$, warm fluid $\left(T^{\prime}>0\right)$ away from the wall, a strong negative correlation in the streamwise direction is expected. Indeed, this is observed in Fig. 17 (top). Interestingly, for the S6 and HS6 cases, an inverted, positive correlation is noted in the center of the channel. In other words, a positive streamwise velocity fluctuation is accompanied by a positive temperature fluctuation away from the wall in the six-strip cases; this is not seen in the three-strip case, where a negative $\left\langle u^{\prime} T^{\prime}\right\rangle$ correlation is maintained throughout the majority of the channel. The fact that an inverted, positive correlation is observed in the six-strip cases can be ascribed to the enhanced mixing that takes place between the hot and cold fluids. Unsurprisingly, the velocitytemperature correlation in the spanwise direction shows a periodic pattern (Fig. 17, bottom); this follows directly from the heating strip alignment.
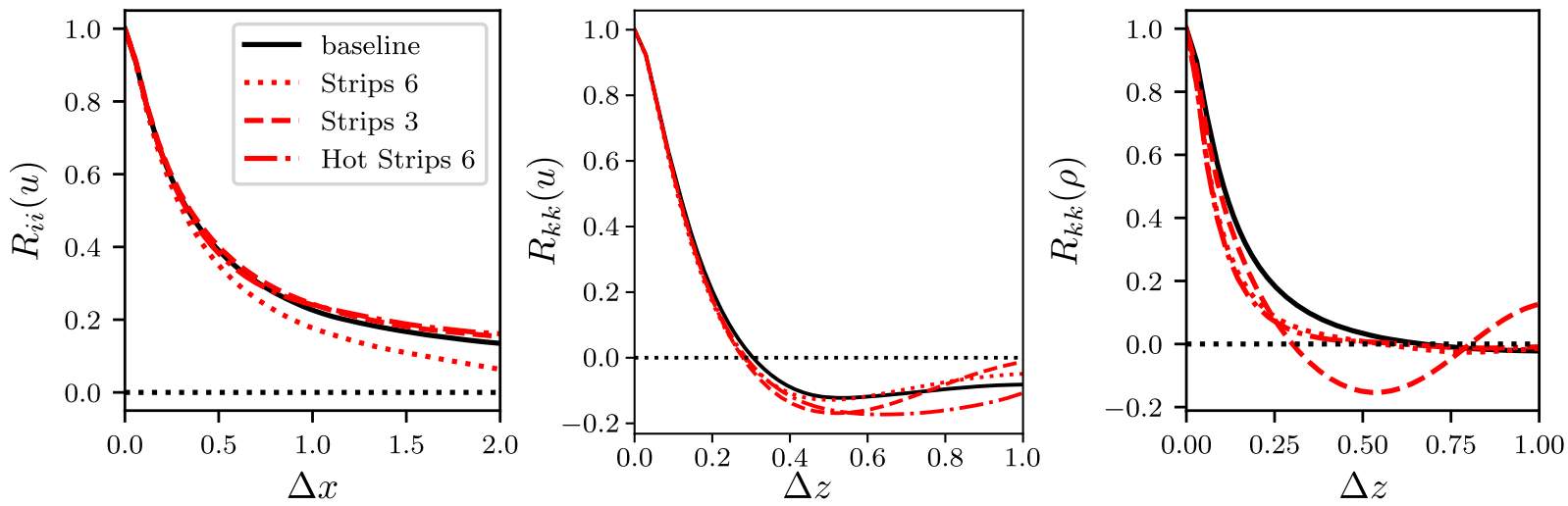

FIG. 16. Two-point correlations at $y / h=0.25$ of $u_{x}$ in the streamwise (left) and spanwise (center) directions. The right pane shows the spanwise density two-point correlations. 

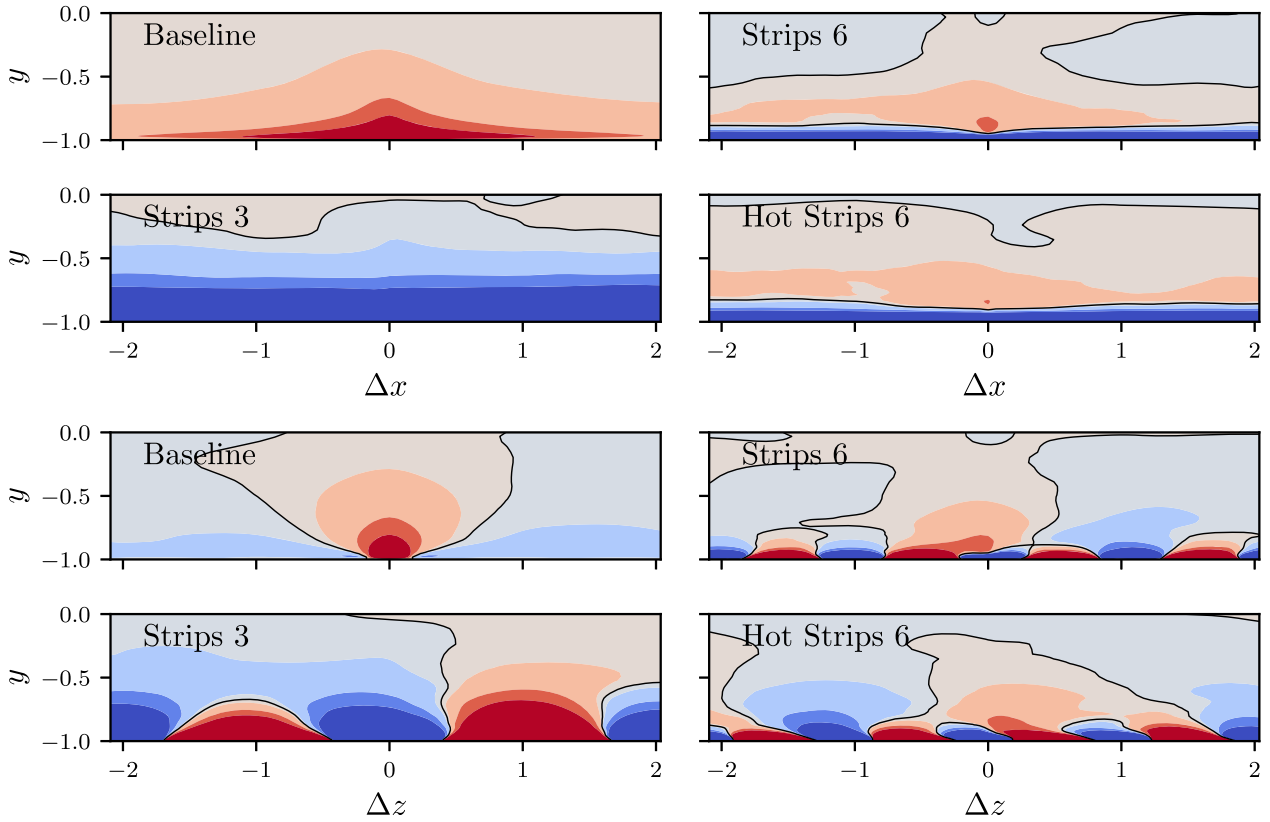

FIG. 17. Normalized two-point correlations of $\left\langle u^{\prime} T^{\prime}\right\rangle$ in the $x$-direction (top) and $z$-direction (bottom).

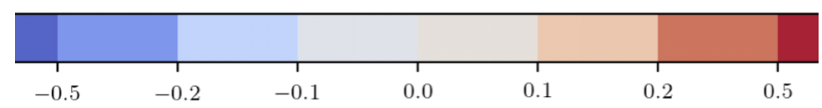

\section{B. Skin friction}

We now turn our attention to the prospect of drag reduction. Note that this should be achieved despite a non-negligible increase in the local viscosity of the fluid at the wall due to the local heating. To quantify the drag reduction potential, we define the skin friction as follows:

$$
C_{f}=\frac{\left\langle\tau_{w}\right\rangle}{\frac{1}{2} \rho U^{2}},
$$

where the denominator represents the dynamic pressure and the numerator is the average shear at the wall,

$$
\left\langle\tau_{w}\right\rangle=\left\langle\left.\mu \frac{d u}{d y}\right|_{w}\right\rangle
$$

In classical, isothermal wall-bounded flows, the right-hand side of Eq. (6) can be simplified to $\left.\langle\mu\rangle \frac{d\langle u\rangle}{d y}\right|_{w}$. In the present case, however, since $\mu$ and $d u / d y$ are correlated (see Fig. 6, for example), such a simplification cannot be assumed. Instead, to evaluate the shear at the wall, one should either directly compute the average of the product of the local viscosity and the velocity gradient [as written in Eq. (6)], or alternatively, calculate the product of the conditionally-averaged velocity gradient and viscosity. Using a well-established lemma from statistics, the skin friction can then be written as follows:

$$
C_{f}=\frac{\left\langle\tau_{w}\right\rangle}{\frac{1}{2} \rho_{0} U^{2}}=\frac{\left\langle\left.\mu \frac{d u}{d y}\right|_{w}\right\rangle}{\frac{1}{2} \rho_{0} U^{2}}=\frac{\left.\langle\mu\rangle \frac{d\langle u\rangle}{d y}\right|_{w}}{\frac{1}{2} \rho_{0} U^{2}}+\frac{\operatorname{cov}\left(\mu,\left.\frac{d u}{d y}\right|_{w}\right)}{\frac{1}{2} \rho_{0} U^{2}},
$$

where brackets $\langle\cdot\rangle$ indicate the expected value (planar-averaged value in our case), and $\operatorname{cov}(\cdot, \cdot)$ represents the covariance of two statistically correlated variables. Equation (7) can be made nondimensional (using the channel half-height, average wall viscosity, and bulk viscosity) as follows:

$$
C_{f}=\left.\frac{2}{R e} \frac{d\langle u\rangle}{d y}\right|_{w}+\frac{2}{R e}\left\langle\left(\frac{\mu}{\langle\mu\rangle}-1\right)\left(\left.\frac{d u^{\prime}}{d y}\right|_{w}\right)\right\rangle,
$$

where the second term on the right-hand side of Eq. (8) is the normalized covariance of the viscosity and the velocity gradient at the wall.

A negative covariance should arise as a higher temperature/viscosity is accompanied by a lower density; this would result in a smaller velocity gradient at the wall. The computed negative covariance [second term of Eq. (8)] represents $-1.388 \%$, $-1.517 \%$, and $-2.341 \%$ of the skin friction for the S6, S3, and HS6 cases, respectively. This represents a non-negligible source of error in the computation of the skin friction calculations for these flows.

To quantify the drag reduction due to the inhomogeneous wall heating, two drag reduction metrics are defined. First, $D R$ is defined as the ratio of the skin friction of the heated cases (S3, S6, or HS6) to that of the unheated, baseline case (UH) as follows:

$$
D R=\frac{C_{f}(\mathrm{~S} 3, \mathrm{~S} 6, \text { or HS6 })}{C_{f}(\mathrm{UH})} .
$$

We recall that we numerically set the bulk momentum in all our cases to the same value given the streamwise-periodic nature of the flow. Therefore, the heating will modify the bulk Reynolds number. To offer a more representative metric, an effective drag reduction, 
TABLE IV. Conditional (based on the hot and cold regions of the channel) and global drag reduction of the streamwise-aligned heating strips.

\begin{tabular}{lcccc}
\hline \hline Drag reduction metric & UH & S6 & S3 & HS6 \\
\hline $\mathrm{DR}_{H O T}$ & 1 & 0.985 & 0.976 & 1.022 \\
$\mathrm{DR} C O L D$ & 1 & 1.003 & 1.027 & 1.004 \\
$\mathrm{DR}$ & 1 & 0.995 & 1.009 & 1.008 \\
$\mathrm{DR}_{H O T}^{*}$ & 1 & 0.952 & 0.942 & 0.953 \\
$\mathrm{DR}_{C O L D}^{*}$ & 1 & 0.965 & 0.991 & 0.935 \\
$\mathrm{DR}^{*}$ & 1 & 0.959 & 0.972 & 0.940 \\
\hline \hline
\end{tabular}

$D R^{*}$, is defined as follows:

$$
D R^{*}=\frac{C_{f}(\mathrm{~S} 3, \mathrm{~S} 6, \text { or HS6 })}{C_{f}^{*}(\mathrm{UH})},
$$

with

$$
C_{f}^{*}=\frac{\left.\left\langle\mu_{\bmod }\right\rangle \frac{d\langle u\rangle}{d y}\right|_{w}}{\frac{1}{2} \rho_{0} U^{2}} .
$$

In Eq. (11), $C_{f}^{*}$ corresponds to a modified skin friction coefficient, defined for the unheated case, with an equivalent viscosity: $\left\langle\mu_{\bmod }\right\rangle$ $=\frac{1}{3} \mu_{\text {hot }}\left(\mathrm{c} \cdot T_{\text {ref }}\right)+\frac{2}{3} \mu_{\text {wall }}\left(T_{\text {ref }}\right)$. In essence, this represents an areaaveraged viscosity (one-third of the wall has the heated strips, twothirds does not), where the constant, $c$, takes on the value of 1.15 or 1.3 (corresponding to $T_{\text {strips }} / T_{\text {wall }}$ ), depending on the heated case. The results of the conditional and global drag reductions, computed using both metrics, are listed in Table IV.

Using targeted heating and with moderate wall temperatures (cases S3 and S6), we note that despite a $4 \%$ increase in the average viscosity at the wall, the effective shear remains nearly unchanged (S3) or even slightly reduced (S6). This, however, does not translate into drag reductions under strongly-heated conditions, for the increased near-wall viscosity counteracts any potential benefit on the turbulence-induced drag. Interestingly, for the moderately-heated cases, an effective drag reduction of $2 \%$ is observed above the heating strips. However, this is canceled out by a larger drag increase in the unheated (cold) regions of the channel. A much more sizable drag reduction potential is obtained when taking into account the equivalent, area-averaged viscosity. Particularly, a maximum drag reduction of $6 \%$ is obtained for HS6. Similarly, when conditionally averaging, a significant drag reduction is observed above the heating strips.

As a final measure of the drag reduction, the individual contributions of turbulence on the skin friction coefficient are identified by decomposing $C_{f}$ using the compressible version of the Fukagata, Iwamoto, and Kasagi (FIK) identity. ${ }^{33}$ The FIK decomposition was initially developed for constant viscosity walls and for incompressible flows. However, it is presented here in a slightly modified form to take into account viscosity variations. Following Gomez et al., ${ }^{34}$

$$
\begin{aligned}
C_{f}= & \underbrace{\frac{12}{R e}}_{C_{L}}+\underbrace{6 \int_{-1}^{0} y\langle\rho\rangle\left\langle u^{\prime \prime} v^{\prime \prime}\right\rangle d y}_{C_{T}}+\underbrace{\frac{12}{R e} \int_{-1}^{0}-y\langle\tilde{\mu}\rangle \frac{\partial\langle u\rangle}{\partial y} d y}_{C_{C}} \\
& +\underbrace{\frac{12}{R e} \int_{-1}^{0}-y\left(\mu^{\prime}\left(\frac{\partial u^{\prime}}{\partial y}+\frac{\partial v^{\prime}}{\partial x}\right)\right) d y}_{C_{C T}} .
\end{aligned}
$$

In Eq. (12), $\langle\tilde{\mu}\rangle$ represents $\langle\mu\rangle-1$, and the double prime indicates a Favre-averaged quantity. The Reynolds number is defined using the bulk quantities of the flow; this accounts for the temperaturedependent viscosity and density.

The skin friction is decomposed into a laminar contribution $\left(C_{L}\right)$, a turbulent contribution $\left(C_{T}\right)$, a compressible contribution $\left(C_{C}\right)$, and a turbulent-compressible contribution $\left(C_{C T}\right)$. At sufficiently high Reynolds numbers, energy-containing motions account for the majority of the turbulent skin friction. ${ }^{25,35}$ Hence, at our Reynolds number of $R e_{\tau} \approx 460$, over $90 \%$ of the skin friction is due to turbulence. Table $V$ lists the various contributions to the skin friction. As previously noted, the optimal heating configuration (S6) results in a reduction of the total skin friction by around $1.2 \%$. It should be noted that our skin friction balance falls within $5 \%$ of the total skin friction computed via Eq. (8); a similar level of error in the FIK balance was noted in Ref. 25.

\section{SUMMARY AND CONCLUSIONS}

The use of streamwise-aligned thermal strips as a means for turbulent flow control and drag reduction is explored in this work. A modest drag reduction is observed, despite a local increase in the near-wall viscosity. On its own, the increase in local viscosity is expected to yield a non-negligible increase in drag. However, due to the presence of the streamwise-aligned heating strips, the near-wall turbulence motion is impeded. This is evidenced by a reduction in the streamwise and spanwise correlation lengths and from changes to the probability distribution of the turbulence sweep and ejection events. The large-scale organization is not significantly altered, as shown by a bidimensional empirical mode decomposition (BEMD) and by conditionally averaged statistics. The results of the above observations lead to an effective drag reduction of $6 \%$ for an optimal heating strip alignment. By decomposing the skin friction using

TABLE V. Decomposition of the contributions to the skin friction based on Gomez et al. ${ }^{34}$

\begin{tabular}{lcccccc}
\hline \hline Case & $C_{L}(\times 100)$ & $C_{T}(\times 100)$ & $C_{C}(\times 100)$ & $C_{C T}(\times 100)$ & Sum & Change \\
\hline UH & 0.03528 & 0.53551 & $4.17708 \times 10^{-6}$ & $1.11574 \times 10^{-8}$ & 0.5708 & $\ldots$ \\
S6 & 0.03484 & 0.52933 & $4.22983 \times 10^{-6}$ & $5.16331 \times 10^{-9}$ & 0.5641 & $-1.2 \%$ \\
S3 & 0.03484 & 0.53633 & $4.24738 \times 10^{-6}$ & $7.00041 \times 10^{-9}$ & 0.5712 & $+0.06 \%$ \\
HS6 & 0.03439 & 0.53664 & $4.29842 \times 10^{-6}$ & $3.16286 \times 10^{-9}$ & 0.5719 & $+0.2 \%$ \\
\hline \hline
\end{tabular}


the FIK identity, it is noted that the turbulence contribution to the skin friction is the main contributor to this drag reduction. Although the greatest relative change in the FIK decomposition is noted in the $C_{L}$ term, the laminar term has a much smaller contribution to the overall drag compared to the turbulence term at these Reynolds numbers.

The work, while exploratory in nature, shows that some benefits may be drawn from a carefully aligned heating array in wallbounded turbulent flows. Under inhomogeneous heating conditions, a local viscosity increase as well as a reduced density can be used to modulate the near-wall turbulent structures. Although the drag reduction potential remains modest, the approach followed can be thought of as being energy neutral, in a sense that recycled waste heat, e.g., from the combustion engine can be used to generate thermal inhomogeneities on the surface of a nacelle. Additionally, readers should bear in mind that the drag reduction is possibly modest due to the use of a gas as the working fluid. Naturally, within a liquid, a more tangible drag reduction is expected. Finally, the study considered only a limited range of heating and spatial variations. More efficient heating arrangements can be envisioned. This is subject to future work. Compared to some of the modern drag reduction approaches, such as spanwise wall oscillations or inhomogeneous porosity, the effectiveness of wall heating as a method to reduce drag appears to be very modest.

\section{ACKNOWLEDGMENTS}

This research was enabled in part by support provided by SciNet (www.scinethpc.ca), Sharcnet (www.sharcnet.ca), and Compute Canada (www.computecanada.ca). The authors would like to acknowledge the support from the Natural Sciences and Engineering Council of Canada (NSERC) through the CGS-M and PGS-D programs.

\section{APPENDIX: EVOLUTION OF HIGHER-ORDER STATISTICS}

The validity of the numerically generated DNS database is assessed in light of the slight drag reduction noted as a result of the local heating to the channel walls. Figure 18 (left) shows the evolution of the velocity gradient at the wall for all the cases studied, whereas Fig. 18 (right) shows the evolution of the root-mean-square
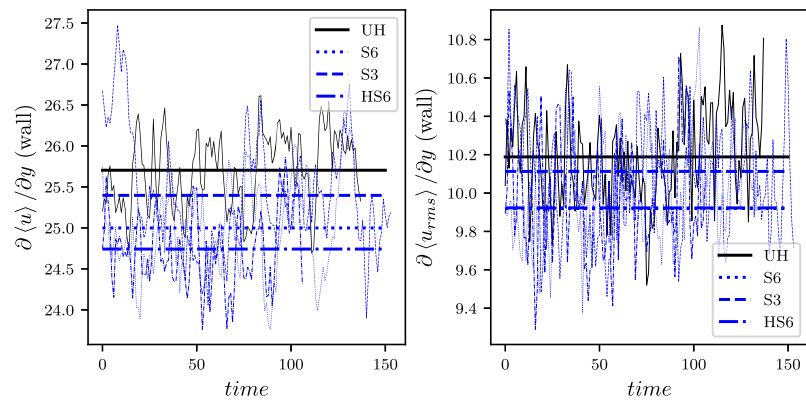

FIG. 18. Time evolution of the velocity gradient at the wall (left) and the root-meansquare (rms) of the velocity gradient at the wall (right). Horizontal lines represent time-averaged values. of the velocity gradient at the wall. As can be seen, the results highlight the statistically steady-state nature of the flow. In addition to that, the claims stated in the main text are also verified by confirming that the findings apply for a smaller sub-sample of the datasets.

\section{REFERENCES}

${ }^{1}$ D. M. Bushnell and K. J. Moore, “Drag reduction in nature," Annu. Rev. Fluid Mech. 23, 65-79 (1991).

${ }^{2}$ R. García-Mayoral and J. Jiménez, "Hydrodynamic stability and breakdown of the viscous regime over riblets," J. Fluid Mech. 678, 317-347 (2011).

${ }^{3}$ D. M. Bushnell and J. N. Hefner, "Riblets," in Viscous Drag Reduction in Boundary Layers, Progress in Astronautics and Aeronautics (AIAA, 1990), pp. 203-261.

${ }^{4}$ H. Choi, P. Moin, and J. Kim, "Direct numerical simulation of turbulent flow over riblets,” J. Fluid Mech. 225, 503-539 (1993).

${ }^{\mathbf{5}}$ S. Raayai-Ardakani and G. H. McKinley, "Geometric optimization of riblettextured surfaces for drag reduction in laminar boundary layer flows," Phys. Fluids 31, 053601 (2019).

${ }^{6}$ A. Pollard, "Passive and active control of near-wall turbulence," Prog. Aeronaut. Sci. 33, 689-708 (1997).

${ }^{7}$ A. Pollard, "Near-wall turbulence control," in Flow Control: Fundamentals and Practices, edited by M. Gad-el Hak, A. Pollard, and J.-P. Bonnet (Springer-Verlag, Berlin, 1998), pp. 431-466.

${ }^{8}$ M. E. Rosti, L. Brandt, and A. Pinelli, "Turbulent channel flow over an anisotropic porous wall-drag increase and reduction," J. Fluid Mech. 842, 381-394 (2018).

${ }^{9}$ A. Silvestri, F. Ghanadi, M. Arjomandi, R. Chin, B. Cazzolato, and A. Zander, "Attenuation of turbulence by the passive control of sweep events in a turbulent boundary layer using micro-cavities,” Phys. Fluids 29, 115102 (2017).

${ }^{10}$ A. Rajappan, K. Golovin, B. Tobelmann, V. Pillutla, Abhijeet, W. Choi, A. Tuteja, and G. H. McKinley, "Influence of textural statistics on drag reduction by scalable, randomly rough superhydrophobic surfaces in turbulent flow," Phys. Fluids 31, 042107 (2019).

${ }^{11}$ O. Mahfoze and S. Laizet, "Skin-friction drag reduction in a channel flow with streamwise-aligned plasma actuators," Int. J. Heat Fluid Flow 66, 83-94 (2017).

${ }^{12}$ M. T. Hehner, D. Gatti, and J. Kriegseis, "Stokes-layer formation under absence of moving parts-a novel oscillatory plasma actuator design for turbulent drag reduction," Phys. Fluids 31, 051701 (2019).

${ }^{13}$ J. Lee, S. Yoon Jung, H. Jin Sung, and T. Zaki, "Effect of wall heating on turbulent boundary layers with temperature-dependent viscosity," J. Fluid Mech. 726, 196225 (2013).

${ }^{14}$ R. K. Cheng and T. T. Ng, "Some aspects of strongly heated turbulent boundary layer flow," Phys. Fluids 25, 1333-1341 (1982).

${ }^{15}$ Y. Kametani and K. Fukagata, "Direct numerical simulation of spatially developing turbulent boundary layer for skin friction drag reduction by wall surfaceheating or cooling," J. Turbul. 13, 1-20 (2012).

${ }^{16}$ M. Gad-El-Hak, Flow Control: Passive, Active, and Reactive Flow Management (Cambridge University Press, Cambridge, 2000).

${ }^{17}$ R. F. Blackwelder and M. Gad-el Hak, "Method and apparatus for reducing turbulent skin friction,” U.S. patent US4932612A (12 June 1990).

${ }^{18}$ M. Gad-el Hak and R. F. Blackwelder, "A drag reduction method for turbulent boundary layers," in 25th AIAA Aerospace Sciences Meeting (Aerospace Sciences Meetings, Reno, NV, 1987).

${ }^{19}$ M. Gad-el Hak and R. F. Blackwelder, "Selective suction for controlling bursting events in a boundary layer," AIAA J. 27, 308-314 (1989).

${ }^{20}$ H. S. Yoon, O. A. El-Samni, and H. H. Chun, "Drag reduction in turbulent channel flow with periodically arrayed heating and cooling strips," Phys. Fluids 18, 025104 (2006).

${ }^{21}$ H. Mamori, K. Fukagata, S. Obi, and J. Hœpffner, "Drag reduction in channel flow by traveling wave-like surface heating/cooling," in TSFP Digital Library Online (Begel House, Inc., 2009). 
${ }^{22}$ D. Floryan and J. M. Floryan, "Drag reduction in heated channels," J. Fluid Mech. 765, 353-395 (2015).

${ }^{23}$ J. M. Floryan, S. Shadman, and M. Z. Hossain, "Heating-induced drag reduction in relative movement of parallel plates," Phys. Rev. Fluids 3, 1-26 (2018).

${ }^{24} \mathrm{H}$. Choi, P. Moin, and J. Kim, "Active turbulence control for drag reduction in wall- bounded flows," J. Fluid Mech. 262, 75-110 (1994).

${ }^{25}$ C. Cheng, W. Li, A. Lozano-Durán, and H. Liu, "Identity of attached eddies in turbulent channel flows with bidimensional empirical mode decomposition," J. Fluid Mech. 870, 1037-1071 (2019).

${ }^{26}$ J. Larsson and S. K. Lele, "Direct numerical simulations of canonical shock/turbulence interaction," Phys. Fluids 21, 1-12 (2009).

${ }^{27}$ J. Larsson, I. Bermejo-Moreno, and S. K. Lele, "Reynolds- and Mach-number effects in canonical shock-turbulence interaction," J. Fluid Mech. 717, 293-321 (2013).

${ }^{28}$ I. Bermejo-Moreno, J. Bodart, J. Larsson, B. M. Barney, J. W. Nichols, and S. Jones, "Solving the compressible Navier-Stokes equations on up to 1.97 million cores and 4.1 trillion grid points," in Proceedings of SC13: International Conference for High Performance Computing, Networking, Storage and Analysis (IEEE Computer Society, New York, NY, 2013) pp. 1-10.
${ }^{29}$ K. Kim, J.-P. Hickey, and C. Scalo, "Pseudophase change effects in turbulent channel flow under transcritical temperature conditions," J. Fluid Mech. 871, 5291 (2019).

${ }^{30}$ E. Johnsen, J. Larsson, A. V. Bhagatwala, W. H. Cabot, P. Moin, B. J. Olsson, P. S. Rawat, S. K. Shankar, B. Sjogreen, H. C. Yee, X. Zhong, and S. K. Lele, "Assessment of high-resolution methods for numerical simulations of compressible turbulence with shock waves," J. Comput. Phys. 229, 1213-1237 (2010).

${ }^{31}$ L. Agostini and M. A. Leschziner, "On the influence of outer large-scale structures on near-wall turbulence in channel flow," Phys. Fluids 26, 075107 (2014).

${ }^{32}$ W. Schoppa and F. Hussain, "Coherent structure generation in near-wall turbulence," J. Fluid Mech. 453, 57-108 (2002).

${ }^{33}$ K. Fukagata, K. Iwamoto, and N. Kasagi, "Contribution of Reynolds stress distribution to the skin friction in wall-bounded flows," Phys. Fluids 14, L73-L76 (2002).

${ }^{34}$ T. Gomez, V. Flutet, and P. Sagaut, "Contribution of Reynolds stress distribution to the skin friction in compressible turbulent channel flows," Phys. Rev. E 79, 035301 (2009).

${ }^{35}$ M. de Giovanetti, Y. Hwang, and H. Choi, "Skin-friction generation by attached eddies in turbulent channel flow," J. Fluid Mech. 808, 511-538 (2016). 